\title{
Theory and application of laser ultrasonic shear wave birefringence measurements to the determination of microstructure orientation in transversely isotropic, polycrystalline graphite materials
}

\author{
Fan W. Zeng ${ }^{a}$, Cristian I. Contescu ${ }^{b}$, Nidia C. Gallego ${ }^{b}$ and James B. Spicer $^{a^{*}}$ \\ ${ }^{a}$ Department of Materials Science and Engineering, Whiting School of Engineering, The Johns \\ Hopkins University, Baltimore, MD 21218 USA \\ ${ }^{\mathrm{b}}$ Carbon and Composites Group, Materials Science and Technology Division, Oak Ridge \\ National Laboratory, Oak Ridge, TN 37831 USA
}

\begin{abstract}
Laser ultrasonic line source methods have been used to study elastic anisotropy in nuclear graphites by measuring shear wave birefringence. Depending on the manufacturing processes used during production, nuclear graphites can exhibit various degrees of material anisotropy related to preferred crystallite orientation and to microcracking. In this study, laser ultrasonic line source measurements of shear wave birefringence on NBG-25 have been performed to assess elastic anisotropy. Laser line sources allow specific polarizations for shear waves to be transmitted - the corresponding wavespeeds can be used to compute bulk, elastic moduli that serve to quantify anisotropy. These modulus values can be interpreted using physical property models based on orientation distribution coefficients and microcrack-modified, single crystal moduli to represent the combined effects of crystallite orientation and microcracking on material anisotropy. Ultrasonic results are compared to and contrasted with measurements of anisotropy based on the coefficient of thermal expansion to show the relationship of results from these techniques.
\end{abstract}

\section{Introduction}

Common materials used in a variety of engineering applications can be described as being polycrystalline. Namely, the material is composed of connected regions that share a common crystal structure and composition but differ in the crystallographic orientation relative to one another. Each of these regions can be referred to as a grain or crystallite. In many polycrystalline materials, crystallite orientations are nearly random and the material can behave isotropically if a statistically significant number of grains are involved in the physical process being investigated.

*Corresponding author. E-mail: spicer@jhu.edu (James B. Spicer) 
Descriptions of materials behavior that include basic physical properties can be quite simple for isotropic materials and, for a large number of engineering materials, these types of descriptions are sufficient for most purposes. However, if the orientations of crystalline regions in the material are not random and there is some type of preferred orientation for the overall population then the material is said to have texture and will behave anisotropically. This is the case for various nuclear grade graphites produced using processing methods that result in preferred crystallite orientation. The anisotropy in these materials can have profound effects on their performance in nuclear reactors where they can serve as critical structural components [1]. Consequently, significant effort has been invested in the measurement of anisotropy in nuclear graphites, but the results of these measurements have generally not been related quantitatively to the underlying texture of the material even though methods for doing so have been demonstrated previously [2]. By contrast, the relationships between texture and material anisotropy in geological materials and metal alloys have been studied extensively [3-7] since the trajectory of seismic waves as well as the behavior of rolled sheet during plastic forming both depend heavily on this anisotropy [8,9]. The most widely used methods for determination of texture in polycrystalline materials involve the use of x-ray diffraction (XRD). These methods generally provide information about the overall crystallographic orientation of material near the surface by interrogating many crystallites in this region and providing some average response related to the overall population [10,11]. Electron, backscatter diffraction (EBSD) is another method that has been used [12]. Owing to the high spatial resolutions that can be achieved using electron microscopy techniques, orientation maps for individual crystallites at the material surface can be created using EBSD. Statistical analysis of these maps can be performed and results related to those obtained using XRD methods. Neutron diffraction has also been used and can provide information not available using either XRD or EBSD techniques since neutrons penetrate through the bulk of a sample and can provide volumetric texture information $[3,5]$.

The material that is the focus of the work presented here is nuclear graphite - a chemically pure form of polycrystalline graphite that has properties favorable for use in nuclear reactors. In polycrystalline graphite, anisotropy can arise not only from the texture - as occurs in other polycrystalline materials such as ceramics and metals - but also from the preferred orientation of microstructural defects. Alignment of non-spherical pores as well as microcracks can influence properties that depend directly on the geometries and population densities for these defects. 
While the measurement of crystallite orientation can be accomplished using diffraction-based techniques, the orientation of defect structures can be more difficult to determine directly especially if whole populations must be characterized. Since these structures can strongly influence the physical properties of the overall material [13], measurement of the anisotropy of bulk properties can provide insight into the preferred orientation of defect structures in the material.

The use of physical properties measurements to characterize anisotropy and infer texture has been used extensively. In particular, the elastic moduli of polycrystalline materials can be readily assessed using ultrasonic methods and these can be directly related to the underlying materials microstructure $[4,6,7,12,14-17]$. If the material anisotropy is dominated by orientation of crystalline regions, then some useful comparisons can be made between diffraction-based measurements and those obtained using ultrasonic techniques [5,12]. In some sense, these comparisons are tests of structure-property relationships since diffraction techniques provide information directly related to preferred orientation while ultrasonics essentially provides modulus-related information. Results of studies that have considered both generally indicate that there is agreement between diffraction and properties results so long as the differences between the measurement techniques are taken into account [18].

For the work reported here, ultrasonic methods have been used to characterize a transverselyisotropic, polycrystalline graphite and measurements have been interpreted within an overall framework that allows for comparison to other physical property measurements. Ultrasound is sensitive to both texture as well as to the presence and orientation of defect structures and, as a result, ultrasonic measurements indicate the combined effects of these on the material moduli. In particular, the technique of shear wave birefringence using laser ultrasonic methods has been used since it minimizes the effects of material inhomogeneity on measured results while effectively capturing and isolating material anisotropy. These ultrasonic measurements provide information that can be interpreted using simple models describing the elastic moduli of polycrystalline materials. In this work, we show how standard physical property descriptions representing the behaviors of single crystal materials (with and without defects) can be combined with a representation of texture (based on the orientation distribution function) to permit development of simple descriptions for the average properties associated with textured, polycrystalline graphite. 


\section{Physical Properties of Textured Polycrystalline Materials}

\subsection{General Background}

Texture in polycrystalline materials generally arises from the techniques that are used during material processing. Rolling, extrusion as well as molding operations can cause slight reorientation and increased alignment of crystalline regions driven by stresses experienced by the material during the forming process [10]. Since single crystal materials are anisotropic, any texture associated with polycrystalline materials will have physical properties that reflect not only the texture but depend directly on the properties of underlying crystal structure of the constituent crystallites. This implies that polycrystalline materials displaying texture do not behave isotropically and, as a result, more involved descriptions than are used for isotropic materials are required to adequately characterize their behaviors. These descriptions must involve the physical properties of the single crystal material and must also include methods for accounting for the overall behavior of the polycrystalline ensemble. In this work, we will focus on the elastic responses and the thermal expansion characteristics, but the overall method used here has been generalized to other material behaviors that can be described using physical property methods. In the following sections, notation conventions vary and have been chosen to effectively convey the major ideas being described. In some cases, notation directly reflects the underlying computation being performed while at other times the notation represents the essential ideas being developed. A uniform notation could have been used for all sections, but would be at the expense of overall clarity. The compromise used here facilitates the presentation while providing essential details that support the overall results of the work.

\subsection{Physical Properties of Single Crystals and Polycrystalline Materials}

The elastic and thermal expansion behaviors of single crystal materials are well-defined using physical property descriptions. Generally, these behaviors are coupled (differences between the isothermal and adiabatic elastic moduli depend on the thermal expansion) and can also occur simultaneously (stress-induced deformations can combine with those produced by changes in temperature). It will be assumed that all behaviors are linear with respect to field variables stress varies linearly with strain for elastic behavior and strain varies linearly with temperature

changes for thermal expansion. This assumption greatly simplifies descriptions of the corresponding physical properties and these can be found in various reference texts [19,20]. 
Unlike single crystal materials, the bulk material properties of a polycrystalline material depend not only on the single crystal properties but also on the relative orientations of individual crystallites that compose the material. Stated differently, the properties of the polycrystalline must somehow reflect the crystalline properties of the constituent material as well as the symmetries of the polycrystalline ensemble. The simplest approach to deriving properties of the aggregate is to compute a directionally-weighted average that incorporates the fractional volume occupied by crystallites of a given orientation along with the value of the property in a particular direction referred to a coordinate system [10]. While this description is conceptually straightforward, another equivalent method for deriving the properties of the polycrystalline material is to employ the orientation distribution function, $f(\boldsymbol{g})$. This scalar function describes the fraction of material in the representative volume of a given orientation, $g$. The orientation can be provided by an ordered set, $(\psi, \theta, \phi)$, corresponding to the symmetric Euler angles. Using $\boldsymbol{g}=(\psi, \theta, \phi)$, the orientation distribution function can be expressed as a series expansion using the generalized spherical harmonic functions as follows:

$$
f(\psi, \theta, \phi)=\sum_{l=0}^{L_{0}^{\prime}} \sum_{m=-l}^{l} \sum_{n=-l}^{l} W_{l m n} Z_{l m n}(\cos \theta) e^{i m \psi} e^{i n \phi}
$$

where $W_{l m n}$ are the orientation distribution coefficients and $Z_{l m n}$ are the Jacobi Polynomials. Similarly, the components of the tensor, $t$, can be expressed in the sample frame of reference such that

$$
\boldsymbol{t}(\psi, \theta, \phi)=\sum_{l=0}^{L_{0}^{\prime \prime}} \sum_{m=-l}^{l} \sum_{n=-l}^{l} \boldsymbol{t}_{l m n} Z_{l m n}(\cos \theta) e^{i m \psi} e^{i n \phi}
$$

where $\boldsymbol{t}_{l m n}$ represent the tensor coefficients for the various components in the sample frame of reference. Using the expressions given in Eqs. (1) and (2) along with the following representation for the average value for of the tensor property, $\bar{t}$ :

$$
\bar{t}=\int \boldsymbol{t}(\boldsymbol{g}) f(\boldsymbol{g}) d \boldsymbol{g}
$$

and noting the orthonormal properties of the generalized spherical harmonics yields the following: 


$$
\overline{\boldsymbol{t}}=4 \pi^{2} \sum_{l=0}^{L_{0}} \sum_{m=-l}^{l} \sum_{n=-l}^{l} \boldsymbol{t}_{l m n} W_{l m n}
$$

where $L_{0}$ takes on the lower value of either $L_{0}^{\prime}$ or $L_{0}^{\prime \prime}$ [10]. This result indicates that the property in the sample frame of reference depends only on the orientation distribution coefficients and the coefficients for the tensor corresponding to the single crystal property. Generally, the higher the point group symmetry of the crystal system for the constituent crystallites or the higher the symmetry of the distribution function or the lower the rank of the tensor property, the lower the number of orientation distribution coefficients that are needed to describe the average property in the polycrystalline material. For the purposes of this work in which we will consider transversely isotropic materials composed of graphite crystallites having hexagonal point group symmetry, only two orientation distribution coefficients are required to describe the average elastic modulus tensor while thermal expansion can be described using a single orientation distribution coefficient.

\subsection{Models for Elastic Response and Thermal Expansion in Polycrystalline Systems}

\subsubsection{Elastic Response}

For textured polycrystalline materials, the constitutive relation for linear elastic response can be expressed as follows:

$$
\sigma_{i j}=\bar{c}_{i j k l} \varepsilon_{k l}
$$

where $\sigma_{i j}$ is the elastic stress tensor, $\varepsilon_{k l}$ is the infinitessimal strain tensor and $\bar{c}_{i j k l}$ is the effective, linear elastic, stiffness tensor. Self-consistent methods are needed to accurately estimate the effective moduli [8], but these methods will not be pursued here. Upper and lower bounds can be obtained by assuming uniform stress or strain conditions which yield the Reuss and Voigt limits for the polycrystalline moduli, respectively. These limits can be computed readily and yield compact forms that can be written directly. Early publications by Morris [22,23] as well as by Sayers [24,25] are useful in constructing these expressions. Following work by Li and Thompson [26], the components of the elastic modulus tensor that relate to the shear birefringence measurements in this work can be expressed as follows:

$$
\bar{c}_{11}^{I}=c_{11}^{I^{0}}+4 \pi^{2}\left[4\left(10^{1 / 2} / 210\right) A_{1}^{I} W_{200}+3\left(2^{1 / 2} / 105\right) B^{I} W_{400}\right]
$$




$$
\begin{aligned}
& \bar{c}_{44}^{I}=c_{44}^{I^{0}}+4 \pi^{2}\left[\left(10^{1 / 2} / 210\right) A_{3}^{I} W_{200}-4\left(2^{1 / 2} / 105\right) B^{I} W_{400}\right] \\
& \bar{c}_{66}^{I}=c_{44}^{I^{0}}+4 \pi^{2}\left[-\left(10^{1 / 2} / 105\right) A_{3}^{I} W_{200}+\left(2^{1 / 2} / 105\right) B^{I} W_{400}\right]
\end{aligned}
$$

where $I=R$ or $V$ depending on whether the Reuss or Voigt values are needed. In these expressions, Voigt two-index notation has been used instead of tensor notation since this decreases the length of various expressions. Also, expressions for $A_{1}^{I}, A_{3}^{I}$ and $B^{I}$ are provided in Appendix A. According to Eqs. (6)-(8), the polycrystalline moduli depend only on the single crystal elastic constants and the orientation distribution coefficients $W_{200}$ and $W_{400}$.

\subsubsection{Thermal Expansion}

Strains, $\varepsilon_{i j}$, associated with a temperature rise, $\theta$, in a textured, polycrystalline material can be expressed as follows:

$$
\varepsilon_{i j}=\bar{\alpha}_{i j}^{I} \theta
$$

where $\bar{\alpha}_{i j}^{I}$ represents the thermal expansion coefficient tensor for the material and $I=R$ or $V$ depending on whether Reuss or Voigt averaging methods are used. Again, estimates for components of the effective thermal expansion tensor should be obtained using methods that have been described elsewhere, but the upper and lower bounds based on the Reuss and Voigt averaging schemes can be expressed quite simply using algebraic expressions.

Following work by Dunn and Ledbetter [27], the thermal expansion coefficients for a transversely isotropic, polycrystalline material using the Reuss averaging process yields:

$$
\bar{\alpha}_{1}^{R}=\left(2 \alpha_{1}+\alpha_{3}\right) / 3-\left[4(2 / 5)^{1 / 2} \pi^{2}\left(\alpha_{3}-\alpha_{1}\right) / 3\right] W_{200}
$$

and

$$
\bar{\alpha}_{3}^{R}=\left(2 \alpha_{1}+\alpha_{3}\right) / 3+\left[8(2 / 5)^{1 / 2} \pi^{2}\left(\alpha_{3}-\alpha_{1}\right) / 3\right] W_{200}
$$

which are the principal components of the thermal expansion tensor. Note that these expressions are fairly simple and indicate that thermal expansion in the polycrystalline system depends solely on the single crystal thermal expansion coefficients and the orientation distribution coefficient $W_{200}$. 
Once again, following work by Dunn and Ledbetter [27], the corresponding results for the thermal expansion coefficients using the Voigt averaging method yields:

$$
\bar{\alpha}_{1}^{V}=\left(2 \alpha_{1}+\alpha_{3}\right) / 3+2\left(\alpha_{1}-\alpha_{3}\right) \gamma / 3-\eta W_{200}
$$

and

$$
\bar{\alpha}_{3}^{V}=\left(2 \alpha_{1}+\alpha_{3}\right) / 3+2\left(\alpha_{1}-\alpha_{3}\right) \gamma / 3-\kappa W_{200}
$$

where expressions for $\gamma, \eta$ and $\kappa$ are provided in Appendix B. Even though these results for the thermal expansion coefficients contain many more terms than those obtained for Reuss averaging, the polycrystalline thermal expansion coefficients still depend solely on the orientation distribution coefficient $W_{200}$ and on the single crystal values for the thermal expansion coefficient and, in this case, the elastic moduli.

\subsubsection{Modified Hill Approximation}

As has been mentioned, the Voigt and Reuss averages for both the elastic moduli and the thermal expansion coefficients represent bounds that might or might not accurately reflect the actual values obtained in polycrystalline materials. For many materials systems, a first estimate beyond these bounds that might be considered is the Hill approximation - the simple average of the Voigt and Reuss values. While the Hill approximation might reasonably hold under many circumstances, it is not clear why this estimate should be preferred to others especially in polycrystalline graphites since the Voigt and Reuss limits differ significantly from one another. For the purposes of this work, a modified Hill approximation will be used where a weighted average of the Voigt and Reuss limits will be used instead of a simple average. For the elastic moduli we will use

$$
\bar{c}_{i j}=r \bar{c}_{i j}^{R}+(1-r) \bar{c}_{i j}^{V}
$$

and for the thermal expansion coefficients

$$
\bar{\alpha}_{i}=r \bar{\alpha}_{i}^{R}+(1-r) \bar{\alpha}_{i}^{V}
$$

where, in both of these expressions, $r$ is the fractional contribution of the Reuss estimate to the overall property and $0 \leq r \leq 1$.

\subsubsection{Determination of Orientation Distribution Coefficients}


First, consider the elastic properties of the material. In this work, we will present shear birefringence measurements that provide values for $\bar{c}_{11}, \bar{c}_{44}$ and $\bar{c}_{66}$. The essential unknowns in the corresponding models for these moduli are $r$ and the orientation distribution coefficients $W_{200}$ and $W_{400}$. Equations (6)-(8) and (14) can be solved for either $W_{200}$ or $W_{400}$ - related quadratic equations and the solution process used in this work are given in Appendix C. Even though the solution for either orientation distribution coefficient can be expressed in a finite, closed form, it is lengthy and will not be given here. The important result is that our measurements provide sufficient information to uniquely solve for the underlying descriptors of the system. Similarly, when thermal expansion is considered, if values for the two components of the corresponding tensor are measured, then $r$ and $W_{200}$ can be determined using the models presented here. A quadratic equation for $W_{200}$ results (also given in Appendix C), but its solution is too lengthy to be shown here.

\section{Elastic and Thermal Expansion Properties of Single Crystal Graphite Containing Microcracks}

During processing of bulk, nuclear graphite materials, microcracks and oriented pores are introduced into the microstructure of the material. These defects are known to affect various behaviors in graphite including the elastic response of the material [28,29], and related effects need to be considered for interpretation of experimental results obtained in this work. There are various approaches to modeling the effects of microcracks on the elastic properties of polycrystalline materials [30-33] especially those containing high crack densities [34] that could account for the effects of microcracks on the elastic moduli of polycrystalline graphite. However, we will choose to modify the single crystal elastic constants to incorporate the effects of microcracks since it is known that these types of defects readily form along planes perpendicular to the axis of symmetry (c-axis) in graphite. This particular approach approximates conditions that hold in nuclear graphite.

\subsection{Single Crystal Elastic Constants - Effect of Oriented Microcracks}

Fortunately, the elastic moduli for hexagonal, single crystal materials containing microcracks perpendicular to the c-axis have been modeled by Schoenberg and Douma [35]. The results they obtained are presented here as follows:

$$
c_{33}^{\prime}=c_{33}\left(1+E_{N}\right)^{-1}, c_{11}^{\prime}=c_{11}-c_{13}^{2}\left[1-\left(1+E_{N}\right)^{-1}\right] / c_{33}, c_{44}^{\prime}=c_{44}\left(1+E_{T}\right)^{-1},
$$




$$
c_{66}^{\prime}=c_{66}, c_{13}^{\prime}=c_{13}\left(1+E_{N}\right)^{-1}, c_{12}^{\prime}=c_{11}^{\prime}-2 c_{66}
$$

where the prime indicates the stiffness tensor component has been modified by the presence of microcracks, $E_{N}=c_{33} Z_{N}$ and $E_{T}=c_{44} Z_{T}$. Here $Z_{N}$ and $Z_{T}$ represent the normal and tangential compliances of an average crack. If the uncracked material is isotropic, these expressions are in general agreement with Zheng under certain conditions [36]. For the purposes of this work, it will be assumed that $Z_{N} \approx Z_{T}$ since Schoenberg and Sayers indicate that this is true for various types of materials [37]. For analysis of experimental results, these microcrack-modified, single crystal elastic constants will be used with all previous models for the elastic properties of textured, polycrystalline materials.

\subsection{Thermal Expansion - Effect of Oriented Microcracks}

Owing to differences between Voigt and Reuss averaging, the effect of microcracks on thermal expansion in polycrystalline materials can manifest itself directly through the thermal expansion coefficients or indirectly through the elastic moduli. According to Eqs. (12) and (13), the Voigt values for components of the thermal expansion tensor depend directly on the single crystal values for elastic moduli owing to elastic constraint effects. Since these are modified by microcracks, it is clear that the thermal expansion of the polycrystalline material will be affected by microcracking even if the single crystal thermal expansion coefficients are not. For the purposes of this work, it will be assumed that the single crystal values for components of the thermal expansion are not directly affected by pores or microcracks since the influence of these microstructural elements on thermal expansion occurs primarily when elastic constraint occurs in polycrystalline systems. This overall approach to interpreting the influence of microstructure on thermal expansion is consistent with work on thermal expansion in a range of materials systems [38-42] and agrees with early work on nuclear graphites by Sutton and Howard [43] and by Jenkins [44].

\section{Experimental: Materials and Methods}

Using laser ultrasonic line source techniques, shear birefringence measurements were made on NBG-25 - a leading candidate as a structural material produced by SGL Carbon for core support in the next generation nuclear reactors [45]. Reports indicate that NBG-25 is an isostatically- 
molded, superfine-grained $(<50 \mu \mathrm{m})$ graphite produced using petroleum-derived coke [45,46]. Preliminary ultrasonic evaluation of this material indicated that it was transversely isotropic. Two ultrasonic samples of NBG-25 having dimensions approximately $50 \mathrm{~mm}$ by $50 \mathrm{~mm}$ by 6 $\mathrm{mm}$ were cut from a larger sample block. These two samples will be referred to as NBG-25-1 and NBG-25-2. The orientations of these samples were chosen so that the directions of ultrasound propagation (along the $6 \mathrm{~mm}$ thickness direction) would be perpendicular to one another in the frame of reference of the original block. One square surface of each sample was prepared using silicon carbide grinding paper; the other square surface was polished to a mirrorlike finish using $1 \mu \mathrm{m}$ diamond paste so that the optical reflectivity was sufficient for ultrasound detection using optical interferometry. Using simple measurements of sample dimensions and mass, the bulk density of the NBG-25 was measured to be $1.85 \mathrm{~g} / \mathrm{cm}^{3}$. It should be noted that the measured volume includes both open and closed pores and this is appropriate for determination of modulus since ultrasound does not distinguish between the two.

Aspects of the graphite microstructure are shown in Fig. 1 where micrographs of the polished sample surface are shown. The image in Fig. 1(a) was acquired using polarized light microscopy and highlights polarization rotation that occurs as a result of light reflection from the sample surface. Crystalline regions of the microstructure that are not aligned with the surface normal will rotate the polarization and produce intensity variations in the acquired image. This type of imaging can quickly provide a qualitative sense of the orientation of crystalline regions in the graphite. A scanning electron microscope (SEM) micrograph of NBG-25 is shown in Fig. 1(b). Image analysis of the polished graphite surface can reveal detail about graphite structure including size, shape, and distribution of pores. Unlike pores, cracks and microcracks are difficult to identify in the SEM images. However, Mrozowski microcracks and other microstructural crack-like features have been observed in nuclear graphites previously using TEM methods [47-50] and these studies have shown that microcracks generally occur between graphitic planes along the crystal c-axis. 

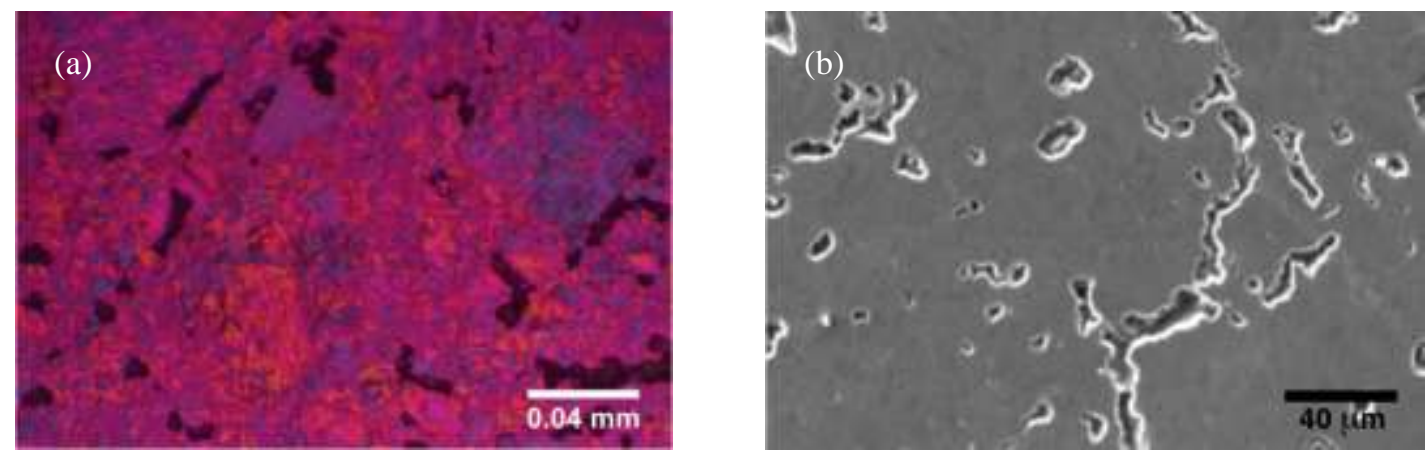

Fig. 1. Micrographs of NBG-25 used in this study. (a) Photomicrograph obtained using polarized light microscopy highlighting regions of varying crystallographic orientation. Black regions are pores. (b) SEM micrograph highlighting pore structure in the graphite.

A schematic of the laser ultrasonic system used to measure shear wave birefringence in these samples is shown in Fig. 2. The output of a Q-switched Nd:YAG laser (pulse duration approximately $10 \mathrm{~ns}$ ) was formed into a line using beam-shaping optics and the pulse energy was adjusted to minimize material removal by ablation. Under these conditions, the laser line source served as a thermoelastic, ultrasonic transmitter that emitted both shear waves as well as longitudinal waves. A Michelson-type, path-stabilized interferometer, located directly opposite the line source in the epicentral position, was used as a receiver. Its effective operating bandwidth was approximately $20 \mathrm{kHz}$ to $50 \mathrm{MHz}$. Owing to the line-like character of the source, it produced shear waves with specific polarization perpendicular to the line direction for components of the displacement field transmitted through the sample thickness. Only shear modes with displacement perpendicular to the laser line are produced since displacements along the line direction are suppressed owing to the symmetry of the source [51]. Figure 3 shows a

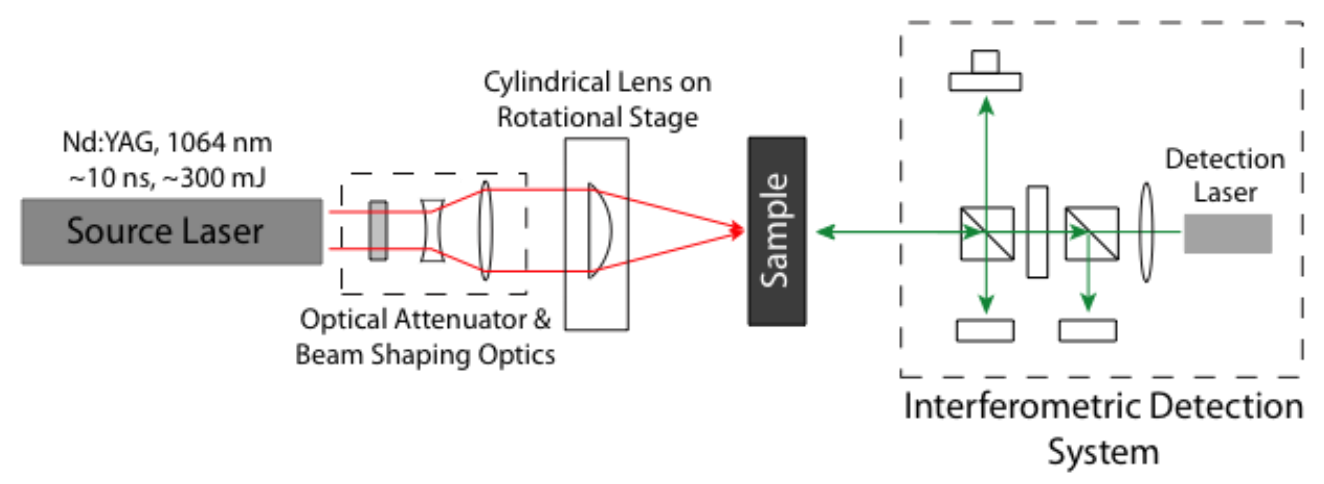

Fig. 2. Schematic of the laser ultrasonic system used to measure shear wave birefringence in graphite. The orientation of the laser line source was changed by mounting the cylindrical lens in a rotational stage. 


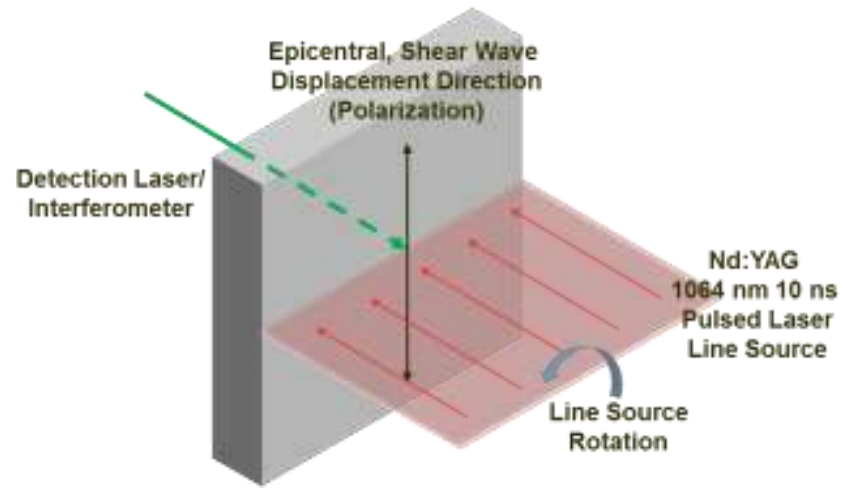

Fig. 3. Schematic of the measurement geometry used to gather ultrasonic waveforms highlighting the shear wave polarization relative to the line source direction. schematic drawing of the measurement configuration where the shear wave propagation direction was fixed while the shear polarization was varied by rotating the orientation of the line source. Timeof-flight measurements were made as a function of the line orientation to assess wavespeed variations as a function of wave polarization. Any wavespeed variations related to polarization

direction can be linked primarily to material anisotropy since shear waves propagate through a fixed volume of material between the source and the receiver. This volume is essentially defined by the receiver spot size on the sample surface (less than $100 \mu \mathrm{m}$ for the work here) as well as the width of the line source (approximately $100 \mu \mathrm{m}$ ). Even though ultrasound arrives at the receiver location from all positions along the line (and encompasses a relatively large volume of material), only wavefront arrivals at the receiver are recorded and these correspond to portions of the ultrasonic field that travel the shortest distances between the line source and the receiver. The lateral resolution provided by the dimensions of the receiver and source permit interrogation of the material on length scales below those typically obtained using traditional ultrasonic techniques. By scanning the source and receiver relative to the sample, variations related to inhomogeneity can be mapped. However, the influence of inhomogeneity on shear wave birefringence measurements is considerably lower than effects attributable to elastic anisotropy since the volume of material interrogated during the ultrasonic measurements is, for the most part, unchanged when signals are recorded for various line orientations. In this work, measurements were made with line orientations of $0^{\circ}$ and $90^{\circ}$ relative to one sample edge. The different line source and sample orientations for the two samples are shown in Fig. 4.

\section{Results and Discussion}


Representative laser ultrasonic waveforms are shown in Fig. 5. In general, the ultrasonic signals from NBG-25-1 have better signal-to-noise characteristics than those from NBG-25-2. Nonetheless, the ultrasonic arrivals for the directly transmitted longitudinal and shear waves are easily identified, and the four different waveforms clearly show the effects of polarization and propagation direction on ultrasonic wavespeeds. These waveforms can be compared to results of modeling studies focused on laser line sources in transversely isotropic systems to assist in the interpretation of arrival times as a function of propagation direction and polarization [52].

In order to assign the recorded waveforms in Fig. 5 to the corresponding measurement geometries depicted in Fig. 4, a few observations regarding expected behaviors in relation to measured results are needed. First, for a transversely isotropic material in which the axis of symmetry is parallel to the direction of ultrasonic propagation, the line source orientation and the corresponding wave polarizations do not affect the ultrasonic wavespeeds. If Cases I and II correspond to this condition, then the orientation of the line source
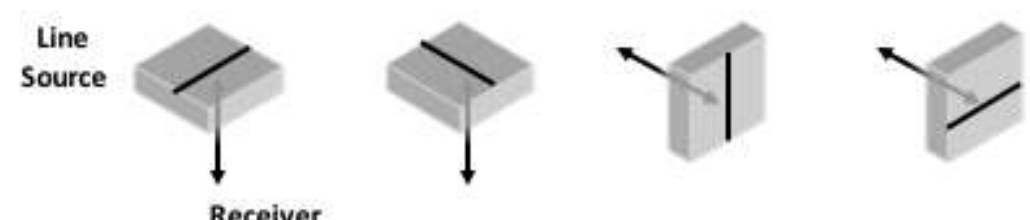

Case 1

Case II

Case III

Case IV

Fig. 4. Illustrations showing relative orientations of the laser line source and the ultrasonic receiver used for assessment of shear birefringence.
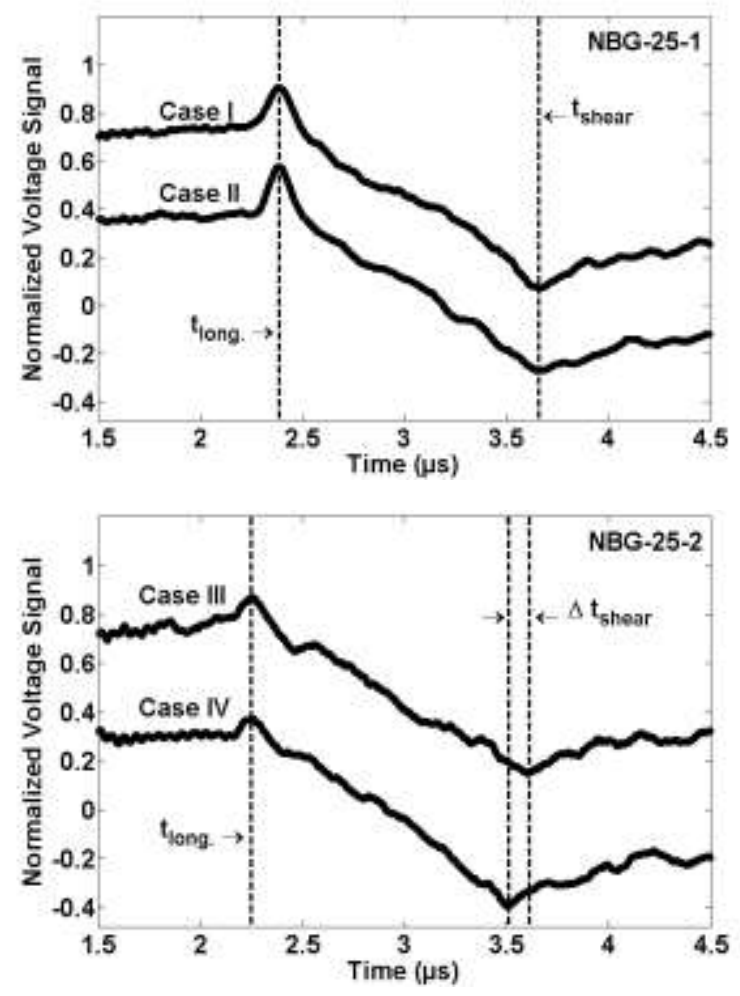

Fig. 5. Laser ultrasonic results obtained on two samples of NBG25 corresponding to the Cases shown in Fig. 4. The sample designated as NBG-25-2 displays shear wave birefringence which is indicated by the difference in shear wave arrival times. Waveforms are shown offset for clarity. 
will not affect the measurement. Results obtained in sample NBG-25-1 appear to approximate this condition. In contrast, if Cases III and IV correspond to propagation perpendicular to the axis of symmetry then the line source orientation will affect the shear wavespeeds leading to shear wave birefringence [52]. This condition appears to be true for the sample NBG-25-2 since the times-of-flight for the shear wave arrivals in this sample differ (indicated by $\Delta t_{\text {Shear }}$ in Fig. $5)$.

These results indicate that NBG-25 could possess symmetries characteristic of a transversely isotropic material. Even so, the limited measurements provided here do not necessarily capture the overall extremes of the elastic anisotropy in this material, and this raises the possibility that the sample reference frames are not necessarily aligned with the material frame of reference. This could be important in a complete assessment of material anisotropy in this system. For the purposes of this work, it will be assumed that results on NBG-25-2 correspond to propagation perpendicular to an axis of symmetry. This is important for analysis of ultrasonic signals and also permits comparison to thermal expansion results presented in the literature for NBG-25.

The models presented for the elastic moduli of transversely isotropic, polycrystalline materials with preferred grain orientation indicate that the components of the elastic stiffness tensor for the bulk material can be expressed using effective isotropic moduli along with perturbations that include the orientation distribution coefficients, $W_{200}$ and $W_{400}$ [26]. These effective isotropic moduli consist of suitable averages of the single crystal modulus values from the stiffness tensor. Based on these models, the ultrasonic waveforms gathered in the orientations represented by Cases III and IV can be used to compute moduli (following procedures outlined in Appendix D) that relate to $W_{200}$ and $W_{400}$. Similarly, it was shown that the thermal expansion coefficients for polycrystalline materials with preferred grain orientation displaying transverse isotropy can be described using effective isotropic coefficients that are suitably modified by terms that depend on the orientation distribution coefficient $\mathrm{W}_{200}$ [27]. Measurements of thermal expansion along and perpendicular to the axis of symmetry are sufficient to infer $W_{200}$ given that the components of the single crystal thermal expansion coefficient tensor as well as the elastic compliances are known. Even though thermal expansion measurements were not performed on the samples used in this study, results from the literature for NBG-25 can be used. 
For a transversely isotropic material, the elastic moduli that can be extracted from the laser ultrasonic measurements indicated for Cases III and IV correspond to $\bar{c}_{11}, \bar{c}_{44}$ and $\bar{c}_{66}$ [52] which were 12.5, 4.84 and 5.13 GPa respectively for the NBG-25 used in this work. Interpreting these results within the framework of a textured, polycrystalline material using microcrackmodified values for the components of the single crystal stiffness tensor along with a modified Hill averaging approach yields values of $W_{200}=0.00196$ and $W_{400}=-0.000757$ for the orientation distribution coefficients. These results were obtained using the following microcrack-modified stiffnesses (all in $c_{11}^{\prime}=1053.79, c_{33}^{\prime}=1.07, c_{44}^{\prime}=0.857, c_{12}^{\prime}=173.79$ and $c_{13}^{\prime}=0.447 \quad$ (corresponding $\quad$ to $\quad$ a microcrack compliance of $\left.Z_{N} \approx Z_{T}=Z=0.5\right)$. These should be compared to the following accepted values for microcrack-free, single crystal graphite (again in GPa) $c_{11}=1060, c_{33}=36.5, c_{44}=4, c_{12}=180$ and $c_{13}=15$. Essentially, elastic constants that depend heavily on microcracks oriented along graphitic planes are significantly modified while others are not altered to any great degree. In addition, a value of $r=0.984$ was produced for the modified Hill averaging scheme indicating that the measured modulus values were close to those associated with the Reuss bound - the lower limit - and this quantitative result is consistent with observations made previously in various graphites [53,54].

Published measurements of the thermal expansion in NBG-25 provide the following values for the components of the thermal expansion coefficient tensor: $\bar{\alpha}_{1}=3.5 \times 10^{-6}{ }^{\circ} \mathrm{C}^{-1}$ and $\bar{\alpha}_{3}=4.2 \times 10^{-6}{ }^{\circ} \mathrm{C}^{-1}$ [55-57]. To interpret these values with the framework for a textured, polycrystalline material, the same microcrack-modified values for the single crystal elastic constants (and the corresponding compliances) were used along with the thermal expansion coefficients for single crystal graphite $\left(\alpha_{1}=-1.2 \times 10^{-6}{ }^{\circ} \mathrm{C}^{-1}\right.$ and $\left.\alpha_{3}=14 \times 10^{-6}{ }^{\circ} \mathrm{C}^{-1}\right)$ [58-63]. Again, the modified Hill averaging approach was used to model the polycrystalline material yielding the orientation distribution coefficient $W_{200}=0.0019$ along with $r=0.973$. Since the orientation distribution coefficients are purely geometrical descriptors of polycrystalline materials, they are independent of the measurement process used to infer them and it should be expected that a value for $W_{200}$ based on measurements of elastic anisotropy should agree with one derived from thermal expansion measurements. Similarly, since the distribution of stresses and strains that occurs among adjoining grains in a material should depend primarily on the 
relative orientations and stiffnesses of the grains and should not differentiate between mechanically-induced or thermally-induced deformations, it should be expected that the fractional contribution of the Reuss and Voigt values to the modified Hill average for the two properties should be the same.

Even so, the agreement of the results for $W_{200}$ and $r$ obtained using ultrasound measurements with those determined using thermal expansion results is remarkable and might appear to be fortuitous. However, the $\sim 3 \%$ difference between the values for $W_{200}$ and the $\sim 1 \%$ difference for the values for $r$ are tied to direct connections between the elastic moduli and thermal expansion that occur in polycrystalline, anisotropic materials since both depend on the single crystal stiffness components [27,63] - the microcrack-modified values in this case. Examination of the expression for these given in Eq. (16) shows that the moduli depend on the normal and tangential compliances of an average microcrack $\left(Z_{N}\right.$ and $Z_{T}$ respectively) where, in this work, these compliances have been assumed to be equal. Essentially, the microcrack compliance becomes an additional parameter that can be varied. While Shoenberg and Douma [35] refer to $Z_{N}$ and $Z_{T}$ as being microcrack compliances, both can be directly related to microcrack densities [36], and, as a result, the role of this additional parameter becomes clear - it reflects the defect content of the graphite. This suggests that changes to $Z_{N} \approx Z_{T}=Z$ could directly relate to changes in the defect content (microcrack density) in the material.

It is possible to use diffraction-based measurements to directly measure orientation distribution coefficients [10,43]. Based on the models developed here, these measurements could be combined with either the elastic or the thermal expansion measurements to derive values for the remaining parameters - including the microcrack density. Doing so presents interesting opportunities for in-service monitoring of graphite integrity. Initial evaluation of the orientation distribution coefficients combined with physical property measurements could be used to establish a baseline for defect content. Changes to the properties would accompany subsequent service-induced changes to defect content and these might be interpreted, within the framework developed in this work, as changes to the microcrack density. Even though any physical property that depends on the presence of microcracks could be used for this type of monitoring, there are advantages to using those that relate more completely to the overall structure of the material. In this work, the focus has been on elastic responses and thermal expansion. Since the 
elastic behavior depends on two orientation distribution coefficients $\left(W_{200}\right.$ and $\left.W_{400}\right)$ instead of only one ( $W_{200}$ as is the case for thermal expansion), measurements of the elastic properties more completely capture the structure-related symmetries of the material than does thermal expansion. Indeed, the general development for the properties of textured materials considered in this work (transversely isotropic, polycrystalline materials composed of grains from the hexagonal crystal system) indicates that any property represented by a second rank tensor (such as thermal expansion) depends only on one orientation distribution coefficient. Other properties such as electrical conductivity, thermal conductivity and dielectric permittivity might be used for characterization or monitoring purposes, but they only partially reflect the underlying structure because they are represented by second rank tensors (for linear systems). While most property measurements incompletely reflect the totality of material structure, those that are represented by higher rank tensors have the potential to capture more structure-related information and this could be important for characterization purposes.

\section{Conclusions}

In this work, an ultrasonic technique for characterization of anisotropy in polycrystalline graphites was demonstrated.

- Laser-based ultrasonics techniques were used to perform shear wave birefringence measurements. These measurements can be used to ultrasonically characterize polycrystalline materials displaying texture and can provide information about the related symmetries of these materials through the elastic moduli.

- Elastic moduli measured using laser ultrasonic methods were interpreted within a framework describing the behaviors of textured anisotropic materials to describe the overall symmetry of a polycrystalline graphite - NBG-25.

- The framework developed to interpret property measurements in polycrystalline ensembles was based on a modified Hill averaging scheme in which both the Reuss and Voigt limits contribute to the overall property average. To successfully apply this averaging scheme to polycrystalline graphite, the effect of microcracking on the elastic response needed to be considered and this was taken into account using microcrack-modified values for the single crystal elastic constants. By incorporating the microcrack-modified values for the single 
crystal elastic constants into the modified Hill average, laser ultrasonic shear wave birefringence measurements could be used to derive values for the orientation distribution coefficients $W_{200}$ and $W_{400}$.

- Thermal expansion measurements on NBG-25 reported in the literature were interpreted using the approach outlined in this work to arrive at a value for $W_{200}$ that agreed well with the corresponding value obtained using ultrasonics measurements.

- Anisotropy in nuclear graphites can be quantified using elasticity and/or thermal expansion measurements, but the elastic response can provide more information about the anisotropy material since it can provide additional texture information through the orientation distribution coefficient $W_{400}$ which cannot be measured using thermal expansion measurements. This additional information about the preferred orientation of graphite microstructure could be useful in forming a more complete understanding of graphite behavior in service environments.

Future work will focus on applying the approach described in this proposal to other polycrystalline graphites to establish quantitative relationships between processing conditions and anisotropy in as-received materials and to investigate variations in anisotropy produced by service-related conditions.

\section{Acknowledgment}

The authors gratefully acknowledge the support of the U.S. Department of Energy (DOE) through the Nuclear Energy University Program (NEUP) Contract No. 00118687. Research at ORNL was performed collaboratively with Johns Hopkins University under the DOE Work for Others (WFO) Program.

\section{References}

[1] BJ Marsden, M Haverty, W Bodel, GN Hall, AN Jones, PM Mummery, M Treifi. Dimensional change, irradiation creep and thermal/mechanical property changes in nuclear graphite, International Materials Reviews, $61 \quad$ (2016) 155-182. DOI:10.1080/09506608.2015.1136460 
[2] T Lokajicek, P Lukas, AN Nikitin, IV Papushkin, VV Sumin, RN Vasin. The determination of the elastic properties of an anisotropic polycrystalline graphite using neutron diffraction and ultrasonic measurements, Carbon. 49 (2011) 1374-1384.

[3] A Allen, M Hutchings, C Sayers, D Allen, R Smith. Use of Neutron-Diffraction Texture Measurements to Establish a Model for Calculation of Ultrasonic Velocities in Highly Oriented Austenitic Weld Material, J.Appl.Phys. 54 (1983) 555-560.

[4] M Hirao, N Hara, H Fukuoka, K Fujisawa. Ultrasonic Monitoring of Texture in Cold-Rolled Steel Sheets, J.Acoust.Soc.Am. 84 (1988) 667-672.

[5] A Clark, R Reno, R Thompson, J Smith, G Blessing, R Fields, P Delsanto, R Mignogna. Texture Monitoring in Aluminum-Alloys - a Comparison of Ultrasonic and NeutronDiffraction Measurement, Ultrasonics. 26 (1988) 189-197.

[6] A Moreau, D Levesque, M Lord, M Dubois, J Monchalin, C Padioleau, J Bussiere. On-line measurement of texture, thickness and plastic strain ratio using laser-ultrasound resonance spectroscopy, Ultrasonics. 40 (2002) 1047-1056.

[7] S Dixon, MP Fletcher, G Rowlands. The accuracy of acoustic birefringence shear wave measurements in sheet metal, J.Appl.Phys. 104 (2008) 114901.

[8] V Vavrycuk. Real ray tracing in anisotropic viscoelastic media, Geophys.J.Int. 175 (2008) 617-626.

[9] RB Thompson. Ultrasonic Characterization of Texture and Formability of Rolled Metal Sheets, Mater.Eval. 51 (1993) 1162-1165.

[10] UF Kocks, CN Tomé, H Wenk, Texture and anisotropy : preferred orientations in polycrystals and their effect on materials properties, Cambridge University Press, Cambridge, U.K. ;New York, 1998.

[11] H-J Bunge, Texture analysis in materials science : mathematical methods, English ed., Butterworths, London ;Boston, 1982.

[12] CL Davis, M Strangwood, M Potter, S Dixon, PF Morris. Prediction of elastic modulus plus anisotropy using X-ray and electron backscattered diffraction texture quantification and ultrasonic (Electromagnetic acoustic transducer) measurements in aluminum sheets, Metallurgical and Materials Transactions A-Physical Metallurgy and Materials Science. 39A (2008) 679-687.

[13] B Kelly. Graphite - the most Fascinating Nuclear Material, Carbon. 20 (1982) 2-11. 
[14] S Sato, S Miyazono. Studies of Elastic Modulus of Irradiated Graphite by an Ultrasonic Pulse Method, Carbon. 2 (1964) 103-114.

[15] T Oku, T Usui, M Eto, Y Fukuda. Effect of Compressive Stress on Youngs Modulus of Unirradiated and Irradiated Nuclear Graphites, Carbon. 15 (1977) 3-8.

[16] T Maruyama, M Eto, T Oku. Elastic-Modulus and Bend Strength of a Nuclear Graphite at High-Temperature, Carbon. 25 (1987) 723-726.

[17] Y Li, R Thompson. Effects of Dispersion on the Inference of Metal Texture from S0 Plate Mode Measurements .1. Evaluation of Dispersion Correction Methods, J.Acoust.Soc.Am. 91 (1992) 1298-1309.

[18] PJ Kielczynski, A Moreau, JF Bussiere. Determination of Texture Coefficients in Hexagonal Polycrystalline Aggregates with Orthorhombic Symmetry using Ultrasounds, J.Acoust.Soc.Am. 95 (1994) 813-827.

[19] JF Nye, Physical properties of crystals: their representation by tensors and matrices, 1984 ed., Clarendon Press ;New York, Oxford Oxfordshire, 1984.

[20] S Haussühl, Physical properties of crystals: an introduction, Wiley-VCH, Weinheim, 2007.

[21] CN Tomé, see Chapter 7 "Tensor Properties of Textured Polycrystals" in Texture and anisotropy : preferred orientations in polycrystals and their effect on materials properties by UF Kocks, CN Tomé, H Wenk, Cambridge University Press, Cambridge, U.K. ;New York, 1998.

[22] PR Morris. Averaging Fourth-Rank Tensors with Weight Functions, J.Appl.Phys. 40 (1969) 447-\&.

[23] PR Morris. Relation of Polycrystalline Elastic Properties to Texture, J.Met. 31 (1979) 8989.

[24] CM Sayers. Angular Dependent Ultrasonic Wave Velocities in Aggregates of Hexagonal Crystals, Ultrasonics. 24 (1986) 289-291.

[25] CM Sayers. The Elastic-Anisotropy of Polycrystalline Aggregates of Zirconium and its Alloys, J.Nucl.Mater. 144 (1987) 211-213.

[26] Y Li, R Thompson. Relations between Elastic-Constants Cij and Texture Parameters for Hexagonal Materials, J.Appl.Phys. 67 (1990) 2663-2665. 
[27] M Dunn, H Ledbetter. Thermal-Expansion of Textured Polycrystalline Aggregates, J.Appl.Phys. 78 (1995) 1583-1588.

[28] DPH Hasselman, Voigt and Reuss Moduli of Polycrystalline Solids with Microcracks, J Am Ceram Soc. 53 (1970) 170.

[29] P Wagner, JA O'Rourke, PE Armstrong. Porosity Effects in Polycrystalline Graphite, J Am Ceram Soc. 55 (1972) 214-219.

[30] JA Hudson. Wave Speeds and Attenuation of Elastic-Waves in Material Containing Cracks, Geophysical Journal of the Royal Astronomical Society. 64 (1981) 133-150.

[31] O Nishizawa. Seismic Velocity Anisotropy in a Medium Containing Oriented Cracks Transversely Isotropic Case, Journal of Physics of the Earth. 30 (1982) 331-347.

[32] JM Assad, RH Tatham, JA McDonald. A Physical Model Study of Microcrack-Induced Anisotropy, Geophysics. 57 (1992) 1562-1570.

[33] F Guerrero, I Sevostianov, A Giraud. On a Possible Approximation of Changes in Elastic Properties of a Transversely Isotropic Material due to an Arbitrarily Oriented Crack, Int.J.Fract. 153 (2008) 169-176.

[34] Y Hu, GA McMechan. Theoretical Elastic Stiffness Tensor at High Crack Density, Journal of Seismic Exploration. 19 (2010) 43-68.

[35] M Schoenberg, J Douma. Elastic Wave-Propagation in Media with Parallel Fractures and Aligned Cracks, Geophys.Prospect. 36 (1988) 571-590.

[36] Z Zheng. Seismic anisotropy due to stress-induced cracks, Int.J.Rock Mech.Min.Sci. 37 (2000) 39-49.

[37] M Schoenberg, CM Sayers. Seismic Anisotropy of Fractured Rock, Geophysics. 60 (1995) 204-211.

[38] DE Bowles. Effect of Microcracks on the Thermal-Expansion of Composite Laminates, J.Composite Mater. 18 (1984) 173-187.

[39] S Ghabezloo. Micromechanical analysis of the effect of porosity on the thermal expansion coefficient of heterogeneous porous materials, Int.J.Rock Mech.Min.Sci. 55 (2012) 97-101.

[40] S Ghabezloo. Effect of porosity on the thermal expansion coefficient: A discussion of the paper 'Effects of mineral admixtures on the thermal expansion properties of hardened cement 
paste' by ZH Shui, R. Zhang, W. Chen, D. Xuan, Constr. Build. Mater. 24 (9) (2010) 17611767, Constr.Build.Mater. 24 (2010) 1796-1798.

[41] Y Kobayashi, M Katayama, M Kato, S Kuramochi. Effect of Microstructure on the Thermal Expansion Coefficient of Sintered Cordierite Prepared from Sol Mixtures, J Am Ceram Soc. 96 (2013) 1863-1868.

[42] E Sicat, F Gong, D Zhang, T Ueda. Change of the Coefficient of Thermal Expansion of Mortar Due to Damage by Freeze Thaw Cycles, Journal of Advanced Concrete Technology. 11 (2013) 333-346.

[43] AL Sutton, VC Howard. The Role of Porosity in the Accommodation of Thermal Expansion in Graphite, J.Nucl.Mater. 7 (1962) 58-71.

[44] GM Jenkins. The Thermal Expansion of Polycrystalline Graphite, J.Nucl.Mater. 13 (1964) 33-39.

[45] TD Burchell, R Bratton, W Windes, NGNP Graphite Selection and Acquisition Strategy, ORNL/TM-2007/153, Oak Ridge National Laboratory, 2007.

[46] M Davies. Graphite core components and the graphite core assembly of a High Temperature Gas-cooled Reactor (HTGR), presented at the Technical Meeting on HighTemperature Qualification of High Temperature Gas Cooled Reactor Materials held by the IAEA in Vienna, Austria, June 10-13, 2014, https://www.iaea.org/NuclearPower/Downloadable/Meetings/2014/2014-06-10-06-13-TMNPTD/20_ASME_Articles_4000-6000.pdf dated May 15, 2013, accessed June 2016.

[47] KY Wen, TJ Marrow, BJ Marsden. The microstructure of nuclear graphite binders, Carbon. 46 (2008) 62-71.

[48] S Chi, G Kim. Comparison of $3 \mathrm{MeV} \mathrm{C}(+)$ ion-irradiation effects between the nuclear graphites made of pitch and petroleum cokes, J.Nucl.Mater. 381 (2008) 98-105.

[49] K Wen, J Marrow, B Marsden. Microcracks in nuclear graphite and highly oriented pyrolytic graphite (HOPG), J.Nucl.Mater. 381 (2008) 199-203.

[50] C Karthik, J Kane, DP Butt, WE Windes, R Ubic. Microstructural Characterization of Next Generation Nuclear Graphites, Microscopy and Microanalysis. 18 (2012) 272-278.

[51] DH Hurley, JB Spicer. Line source representation for laser-generated ultrasound in an elastic transversely isotropic half-space, J.Acoust.Soc.Am. 116 (2004) 2914-2922. 
[52] JB Spicer, FW Zeng. Line source representations for shear wave birefringence measurements in transversely isotropic materials using laser ultrasonics, Wave Motion. 61 (2016) 1-10.

[53] JR Cost, KR Janowski, RC Rossi. Elastic Properties of Isotropic Graphite, Philosophical Magazine. 17 (1968) 851-854.

[54] JB Spicer, LR Olasov, FW Zeng, K Han, NC Gallego, CI Contescu. Laser ultrasonic assessment of the effects of porosity and microcracking on the elastic moduli of nuclear graphites, J.Nucl.Mater. 471 (2016) 80-91.

[55] A Vreeling, J van der Laan, D D'Hulst, P ten Pierick, R den Boef, F van den Berg, Graphite Irradiation for the European HTR-M/MI Project, Nuclear Research and Consultancy Group, ftp://ftp.nrg.eu/pub/www/nrg/2004composites/NRG_Vreeling.pdf accessed June 2016, N.D.

[56] JA Vreeling, O Wouters, JG van der Laan. Graphite irradiation testing for HTR technology at the High Flux Reactor in Petten, J.Nucl.Mater. 381 (2008) 68-75.

[57] G Vasudevamurthy, TS Byun, P Pappano, LL Snead, TD Burchell. Effect of specimen size and grain orientation on the mechanical and physical properties of NBG-18 nuclear graphite, J.Nucl.Mater. 462 (2015) 1-7.

[58] F Entwisle. Thermal Expansion of Pyrolytic Graphite, Physics Letters. 2 (1962) 236-238.

[59] WH Martin, F Entwisle. Thermal Expansion of Graphite Over Different Temperature Ranges, J.Nucl.Mater. 10 (1963) 1-7.

[60] WC Morgan. Thermal Expansion Coefficients of Graphite Crystals, Technical Report BNWL-SA-3838, Battelle-Northwest Laboratory, Richland, Washington, 1971.

[61] WC Morgan. Thermal-Expansion Coefficients of Graphite Crystals, Carbon. 10 (1972) 73-79.

[62] AC Bailey, B Yates. Anisotropic Thermal Expansion of Pyrolytic Graphite at Low Temperatures, J.Appl.Phys. 41 (1970) 5088-5091.

[63] DKL Tsang, BJ Marsden, SL Fok, G Hall. Graphite thermal expansion relationship for different temperature ranges, Carbon. 43 (2005) 2902-2906. 


\section{Appendix A: Additional Terms for Polycrystalline Elastic Response}

These are expressions to be used in models based on those developed by Li and Thompson [26] for the elastic moduli of polycrystalline materials composed of grains with hexagonal crystal symmetry. For the Voigt model,

$$
\begin{aligned}
& c_{11}^{0^{V}}=\left(8 c_{11}+3 c_{33}+4 c_{13}+8 c_{44}\right) / 15 \\
& c_{44}^{0^{V}}=\left(7 c_{11}-5 c_{12}+2 c_{33}-4 c_{13}+12 c_{44}\right) / 30 \\
& c_{12}^{0^{V}}=\left(c_{11}+5 c_{12}+c_{33}+8 c_{13}-4 c_{44}\right) / 15 \\
& A_{1}^{V}=\left(4 c_{11}-3 c_{33}-c_{13}-2 c_{44}\right) \\
& A_{3}^{V}=\left(-5 c_{11}+7 c_{12}+2 c_{33}-4 c_{13}+6 c_{44}\right) \\
& B^{V}=\left(c_{11}+c_{33}-2 c_{13}-4 c_{44}\right) .
\end{aligned}
$$

For the Reuss model,

$$
\begin{aligned}
& c_{11}^{0^{R}}=\left(s_{11}^{0}+s_{12}^{0}\right) /\left[\left(s_{11}^{0}-s_{12}^{0}\right)\left(s_{11}^{0}+2 s_{12}^{0}\right)\right] \\
& c_{12}^{0^{R}}=-s_{12}^{0} /\left[\left(s_{11}^{0}-s_{12}^{0}\right)\left(s_{11}^{0}+2 s_{12}^{0}\right)\right] \\
& c_{44}^{0^{R}}=1 / s_{44}^{0}
\end{aligned}
$$

where

$$
\begin{aligned}
& s_{11}^{0}=\left(8 s_{11}+3 s_{33}+4 s_{13}+2 s_{44}\right) / 15 \\
& s_{44}^{0}=2\left(7 s_{11}-5 s_{12}+2 s_{33}-4 s_{13}+3 s_{44}\right) / 15 \\
& s_{12}^{0}=\left(s_{11}+5 s_{12}+s_{33}+8 s_{13}-s_{44}\right) / 15 .
\end{aligned}
$$

Also,

$$
A_{1}^{R}=-4\left(c_{44}^{0^{R}}\right)^{2} a_{1}-14 c_{12}^{0^{R}} c_{44}^{0^{R}} a_{0}
$$




$$
\begin{aligned}
& A_{3}^{R}=-4\left(c_{44}^{0^{R}}\right)^{2} a_{3} \\
& B^{R}=-4\left(c_{44}^{0^{R}}\right)^{2} a_{4}
\end{aligned}
$$

where

$$
\begin{aligned}
& a_{0}=\left(s_{11}+s_{12}-s_{33}-s_{13}\right) \\
& a_{1}=\left(4 s_{11}-3 s_{33}-s_{13}-s_{44} / 2\right) \\
& a_{3}=\left(-5 s_{11}+7 s_{12}+2 s_{33}-4 s_{13}+3 s_{44} / 2\right) \\
& a_{4}=s_{11}+s_{33}-2 s_{13}-s_{44} .
\end{aligned}
$$

The single crystal compliances are related to the elastic moduli as follows:

$$
\begin{aligned}
& s_{11}=\left(c_{33} / c_{0}^{2}+\left(c_{11}-c_{12}\right)^{-1}\right) / 2 \\
& s_{33}=\left(c_{11}+c_{12}\right) / c_{0}^{2} \\
& S_{12}=\left(c_{33} / c_{0}^{2}-\left(c_{11}-c_{12}\right)^{-1}\right) / 2 \\
& S_{13}=-c_{13} / c_{0}^{2} \\
& s_{44}=1 / c_{44}
\end{aligned}
$$

where $c_{0}^{2}=c_{33}\left(c_{11}+c_{12}\right)-2 c_{13}^{2}$.

\section{Appendix B: Additional Terms for Polycrystalline Thermal Expansion}

The expressions provided here are used with models developed from those presented by Dunn and Ledbetter [27] for the thermal expansion of polycrystalline materials composed of grains with hexagonal crystal symmetry.

$$
\begin{aligned}
& \gamma=\left(s_{11}+s_{12}-s_{13}-s_{33}\right) /\left(s_{11}+s_{12}-4 s_{13}+2 s_{33}\right) \\
& \eta=\left[\frac{(2 / 5)^{1 / 2} \pi^{2}\left(\alpha_{3}-\alpha_{1}\right)}{21}\right]\left[\frac{\left(S_{11}^{0}+2 S_{12}^{0}\right)\left[2 A_{1}\left(-7 S_{11}^{0}+S_{12}^{0}\right)+A_{2}\left(S_{11}^{0}-13 S_{12}^{0}\right)\right]}{s_{11}+s_{12}-s_{13}-s_{33}}\right]
\end{aligned}
$$




$$
\kappa=\left[\frac{2(2 / 5)^{1 / 2} \pi^{2}\left(\alpha_{3}-\alpha_{1}\right)}{21}\right]\left[\frac{\left(S_{11}^{0}+2 S_{12}^{0}\right)\left[2 A_{1}\left(4 S_{11}^{0}-7 S_{12}^{0}\right)-A_{2}\left(7 S_{11}^{0}-S_{12}^{0}\right)\right]}{S_{11}+S_{12}-S_{13}-S_{33}}\right]
$$

where

$$
\begin{gathered}
S_{11}^{0}=12 s_{44}\left[s_{33}\left(s_{11}+s_{12}\right)-2 s_{13}^{2}\right]\left[s_{13}^{2}\left(2 s_{11}-2 s_{12}+s_{44}\right)-s_{33}\left(s_{11}^{2}-s_{12}^{2}\right)\right] \\
\times\left[12 s_{13}\left(s_{11}-s_{12}\right)-4\left(s_{11}^{2}-s_{12}^{2}\right)-s_{33}\left(9 s_{11}-5 s_{12}\right)\right] / D_{1} \\
S_{12}^{0}=-12 s_{44}\left[s_{33}\left(s_{11}+s_{12}\right)-2 s_{13}^{2}\right]\left[s_{13}^{2}\left(2 s_{11}-2 s_{12}+s_{44}\right)-s_{33}\left(s_{11}^{2}-s_{12}^{2}\right)\right] \\
\times\left[8 s_{13}\left(s_{11}-s_{12}\right)-\left(s_{11}^{2}-s_{12}^{2}\right)-s_{33}\left(s_{11}-5 s_{12}\right)\right] / D_{1}
\end{gathered}
$$

with

$$
\begin{gathered}
D_{1}=2 s_{13}\left(6 s_{13}-s_{44}\right)\left(2 s_{11}-2 s_{12}+s_{44}\right)-2\left(s_{11}^{2}-s_{12}^{2}\right)\left(6 s_{33}+s_{44}\right) \\
+s_{44}\left(2 s_{13} s_{44}+7 s_{11} s_{33}-5 s_{12} s_{33}\right)
\end{gathered}
$$

and

$$
\begin{gathered}
A_{1}=\left\{2 s_{12}\left(s_{12} s_{33}-2 s_{13}^{2}\right)-s_{11}^{2}\left(2 s_{33}+3 s_{44}\right)+s_{44}\left(3 s_{12}-4 s_{13}\right)\left(s_{12}+s_{13}\right)\right. \\
\left.+s_{11}\left[4 s_{13}^{2}+s_{44}\left(s_{13}+4 s_{33}\right)\right]\right\} / D_{2} \\
A_{2}=\left\{4 s_{12}\left(s_{12} s_{33}-2 s_{13}^{2}\right)+s_{11}^{2}\left(s_{44}-4 s_{33}\right)+s_{44}\left[s_{12}\left(7 s_{33}-s_{12}\right)+s_{13}\left(5 s_{12}-8 s_{13}\right)\right]\right. \\
\left.+s_{11}\left[8 s_{13}^{2}+s_{44}\left(s_{33}-5 s_{13}\right)\right]\right\} / D_{2}
\end{gathered}
$$

with

$$
D_{2}=s_{44}\left(s_{11}-s_{12}\right)\left[s_{33}\left(s_{11}+s_{12}\right)-2 s_{13}^{2}\right] .
$$

\section{Appendix C: Equations for Orientation Distribution Coefficients}

First, the quadratic equations produced for the orientation distribution coefficient, $\boldsymbol{W}_{400}$, using expressions for the material elastic moduli are developed here. Equations (6)-(8) can be expressed in the following form:

$$
\bar{c}_{i i}^{I}=c_{i i}^{0^{I}}+\left[U_{i i}^{I} W_{200}+V_{i i}^{I} W_{400}\right]
$$

where no summation is implied by the repeated subscript. According to Eq. (14), these expressions for the average moduli can be used to construct expressions that represent measured values for $\bar{c}_{11}, \bar{c}_{44}$ and $\bar{c}_{66}$. Anyone of these can be used to solve for $r$ and the remaining two 
can be used to form expressions for the orientation distribution coefficient, $W_{400}$, in terms of $W_{200}$. The following equations were used to develop the results presented in this work:

$$
\begin{aligned}
& 0=W_{400}^{2}\left(V_{11}^{R} V_{66}^{V}-V_{66}^{R} V_{11}^{V}\right)+ \\
& W_{400}\left[\left(\bar{c}_{11}-c_{11}^{0^{V}}-U_{11}^{V} W_{200}\right) V_{66}^{R}-\left(\bar{c}_{11}-c_{11}^{0^{R}}-U_{11}^{R} W_{200}\right) V_{66}^{V}-\right. \\
& \left.\left(\bar{c}_{66}-c_{66}^{0^{V}}-U_{66}^{V} W_{200}\right) V_{11}^{R}+\left(\bar{c}_{66}-c_{66}^{0^{R}}-U_{66}^{R} W_{200}\right) V_{11}^{V}\right]+ \\
& {\left[\bar{c}_{11}\left(c_{66}^{0^{R}}-c_{66}^{0^{V}}+\left(U_{66}^{R}-U_{66}^{V}\right) W_{200}\right)-\bar{c}_{66}\left(c_{11}^{0^{R}}-c_{11}^{0^{V}}+\left(U_{11}^{R}-U_{11}^{V}\right) W_{200}\right)-\right.} \\
& \left.\left(c_{11}^{0^{V}}+U_{11}^{V} W_{200}\right)\left(c_{66}^{0^{R}}+U_{66}^{R} W_{200}\right)+\left(c_{66}^{0^{V}}+U_{66}^{V} W_{200}\right)\left(c_{11}^{0^{R}}+U_{11}^{R} W_{200}\right)\right] \\
& 0=W_{400}^{2}\left(V_{44}^{R} V_{66}^{V}-V_{66}^{R} V_{44}^{V}\right)+ \\
& W_{400}\left[\left(\bar{c}_{44}-c_{44}^{0^{V}}-U_{44}^{V} W_{200}\right) V_{66}^{R}-\left(\bar{c}_{44}-c_{44}^{0^{R}}-U_{44}^{R} W_{200}\right) V_{66}^{V}-\right. \\
& \left.\left(\bar{c}_{66}-c_{66}^{0^{V}}-U_{66}^{V} W_{200}\right) V_{44}^{R}+\left(\bar{c}_{66}-c_{66}^{0^{R}}-U_{66}^{R} W_{200}\right) V_{44}^{V}\right]+ \\
& {\left[\bar{c}_{44}\left(c_{66}^{0^{R}}-c_{66}^{0^{V}}+\left(U_{66}^{R}-U_{66}^{V}\right) W_{200}\right)-\bar{c}_{66}\left(c_{44}^{0^{R}}-c_{44}^{0^{V}}+\left(U_{44}^{R}-U_{44}^{V}\right) W_{200}\right)-\right.} \\
& \left.\left(c_{44}^{0^{V}}+U_{44}^{V} W_{200}\right)\left(c_{66}^{0^{R}}+U_{66}^{R} W_{200}\right)+\left(c_{66}^{0^{V}}+U_{66}^{V} W_{200}\right)\left(c_{44}^{0^{R}}+U_{44}^{R} W_{200}\right)\right]
\end{aligned}
$$

Even though $W_{200}$ could be eliminated from these equations, the solution process followed here treated it as a parameter that was fixed when a specific value for $W_{200}$ yielded values for $W_{400}$ from the solutions for Eqs. (C.2) and (C.3) agreed.

Next, the quadratic equation for $W_{200}$ developed from thermal expansion results will be presented. Equations (10)-(13) can be expressed in the following form:

$$
\bar{\alpha}_{i}^{I}=\alpha^{0^{I}}+X_{i}^{I} W_{200}
$$

According to Eq. (15), these expressions for the average thermal expansion coefficient can be used to construct expressions that represent measured values for $\bar{\alpha}_{1}$ and $\bar{\alpha}_{3}$. Either of these can be used to solve for $r$ and the remaining equation can be used to form an expression for the orientation distribution coefficient $W_{200}$. The following result was used in this work:

$$
\begin{aligned}
& 0=W_{200}^{2}\left(X_{1}^{R} X_{3}^{V}-X_{3}^{R} X_{1}^{V}\right)+ \\
& W_{200}\left[\left(\bar{\alpha}_{1}\left(X_{3}^{R}-X_{3}^{V}\right)-\bar{\alpha}_{3}\left(X_{1}^{R}-X_{1}^{V}\right)-\alpha^{0^{V}}\left(X_{3}^{R}-X_{1}^{R}\right)+\alpha^{0^{R}}\left(X_{3}^{V}-X_{1}^{V}\right)\right]+.\right. \\
& \quad\left(\alpha^{0^{R}}-\alpha^{0^{V}}\right)\left(\bar{\alpha}_{1}-\bar{\alpha}_{3}\right)
\end{aligned}
$$

\section{Appendix D: Relationship of Ultrasonic Measurements to Elastic Moduli}

Using standard transmit-and-receive, through-thickness measurement geometries in which an ultrasonic transmitter is located directly opposite a receiver on a sample surface, the ultrasonic 
wavespeed for a particular wave polarization and propagation direction, $v_{i j}$, can be computed using measurements of the corresponding wave transit time (or time-of-flight), $\Delta t_{i j}$, and the sample thickness, $T$. With an additional measurement of the sample density, $\rho$, the modulus of the material can be determined as follows:

$$
\bar{c}_{i j}=\rho\left(T / \Delta t_{i j}\right)^{2}=\rho v_{i j}^{2}
$$

where $\bar{c}_{i j}$ is the modulus being measured. This expression provides the connection between the models for elastic modulus developed in previous sections to the ultrasonic measurements that were performed to assess shear wave birefringence. 


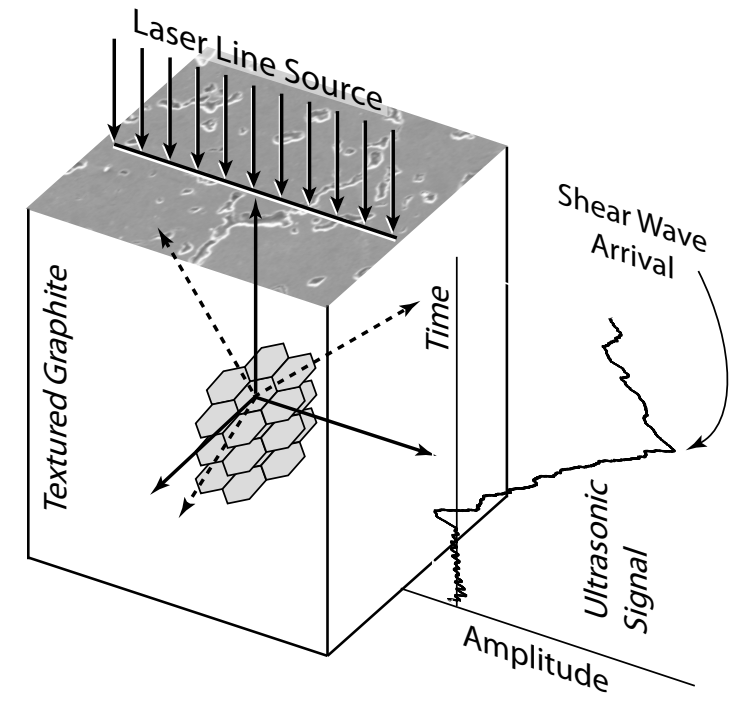

